\title{
TAMENESS AND LOCAL NORMAL BASES FOR OBJECTS OF FINITE HOPF ALGEBRAS
}

\author{
LINDSAY N. CHILDS AND SUSAN HURLEY
}

\begin{abstract}
Let $R$ be a commutative ring, $S$ an $R$-algebra, $H$ a Hopf $R$ algebra, both finitely generated and projective as $R$-modules, and suppose $S$ is an $H$-object, so that $H^{*}=\operatorname{Hom}_{R}(H, R)$ acts on $S$ via a measuring. Let $I$ be the space of left integrals of $H^{*}$. We say $S$ has normal basis if $S \cong H$ as $H^{*}$ modules, and $S$ has local normal bases if $S_{p} \cong H_{p}$ as $H_{p}^{*}$-modules for all prime ideals $p$ of $R$. When $R$ is a perfect field, $H$ is commutative and cocommutative, and certain obvious necessary conditions on $S$ hold, then $S$ has normal basis if and only if $I S=R=S^{H^{*}}$. If $R$ is a domain with quotient field $K, H$ is cocommutative, and $L=S \otimes_{R} K$ has normal basis as $\left(H^{*} \otimes K\right)$-module, then $S$ has local normal bases if and only if $I S=R=S^{H^{*}}$.
\end{abstract}

Suppose $K$ is a number field with ring of integers $R, L$ is a finite Galois extension of $K$ with Galois group $G$, and $S$ is the integral closure of $R$ in $L$. Then $G$ acts as a group of automorphisms of $S$. Relative Galois module theory seeks to understand $S$ as an $R G$-module via this action. The most basic question is to inquire whether $S$ is locally isomorphic to $R G$, that is, for all primes $p$ of $R, S_{p}$ has a local normal basis as a free $R_{p}$-module. This question was answered by Emmy Noether, who showed that $S$ has a local normal basis at every prime $p$ of $R$ if and only if $L / K$ is tamely ramified. Here, tamely ramified means that at each prime ideal $p$ of $R$, the ramification index of any prime $P$ of $S$ lying over $p$ is relatively prime to the residue field characteristic. It is well known that this latter condition is equivalent to the surjectivity of the trace map $\operatorname{tr}: S \rightarrow R, \operatorname{tr}(s)=\sum_{\sigma \in G} \sigma(s)$.

The purpose of this paper is to formulate and prove an extension of Noether's theorem to objects of finite cocommutative Hopf algebras.

Assume now only that $R$ is a commutative ring, $H$ is a Hopf $R$-algebra which is a finitely generated projective $R$-module, and $H^{*}=\operatorname{Hom}_{R}(H, R)$, the dual Hopf algebra. A commutative $R$-algebra $S$ is an $H$-object if $S$ is a right $H$-comodule via a map $\alpha: S \rightarrow S \otimes H$ which is an $R$-algebra homomorphism. If $S$ is an $H$-object, then $\alpha$ induces a measuring $\alpha^{*}: H^{*} \otimes S \rightarrow S$ in the sense of Sweedler [21].

Galois module theory in this setting is the study of $S$ as an $H^{*}$-module via the measuring $\alpha^{*}$. The "trivial" example is $S=H$ itself, with $\alpha=\Delta$, the comultiplication on $H$. So we say that the $H$-object $S$ has normal basis if $S \cong H$ as $H^{*}$-modules, and $S$ has local normal bases if for all primes $p$ of $R, S_{p} \cong H_{p}$ as $H_{p}^{*}$-modules.

Since $R G \cong(R G)^{*}$ as $R G$-modules for $G$ any finite group, this notion of normal basis, when specialized to $H^{*}=R G$, is equivalent to the classical notion.

Received by the editors May 7, 1985 and, in revised form, January 7, 1986.

1980 Mathematics Subject Classification (1985 Revision). Primary 13B05; Secondary 16A24. 
Noether's result in the classical situation says that $S / R$ has local normal bases if and only if the trace map is onto. The trace map is the result of acting by $\sum \sigma$, a generator of the space of integrals of the Hopf algebra $R G$. Thus in seeking an extension of Noether's theorem to an $H$-object $S$, a natural condition to consider is that if $I$ is the space of integrals of $H^{*}$, then $I S=R$. We call an $H$-object $S$ tame if the induced $H^{*}$-module action on $S$ is faithful, $\operatorname{rank}_{R} S=\operatorname{rank}_{R} H$, and $I S=R$.

Our main results are that if the Hopf algebra $H$ is commutative and cocommutative and $S$ is an $H$-object with $S^{H^{*}}=R$, then tameness of $S$ is necessary and sufficient for $S$ to have a normal basis when $R$ is a perfect field, and is necessary and sufficient for $S$ to have local normal bases when $R$ is an integral domain with perfect quotient field $K$. These results are valid without the commutativity assumption on $H$ if $K$ has characteristic zero.

For $H$-objects where $H$ is cocommutative, these results generalize both Noether's theorem and Kreimer and Cook's result $[\mathbf{1 5}]$ that Galois $H$-objects have local normal bases.

The paper is organized as follows.

The first two sections of the paper are devoted to basic properties of tame $H$ objects. We show that if $S$ is an $H$-object which is Galois or which has local normal bases, then $S$ is a tame $H$-object.

The next two sections contain the characterization of $H$-objects with normal basis over a perfect field, when $H$ is commutative and cocommutative. The proof is very "abelian" in the sense that it uses the etale-connected decomposition of finite abelian group schemes over perfect fields.

The final section extends the characterization of $H$-objects with normal basis over fields to a similar characterization of $H$-objects with local normal bases over integral domains.

The basic references for this work are Sweedler [21] for the theory and notation of Hopf algebras, and Chase and Sweedler [7] for the theory of $H$-objects and, in particular, Galois $H$-objects.

Unadorned tensor products are over $R$.

Much of this work is contained in the second author's doctoral dissertation [13]. We wish to thank the referee for numerous insightful suggestions.

1. Preliminaries. We begin by collecting the definitions and results on Hopf algebras which we will need.

Throughout, $R$ is a commutative ring with unity.

(1.1) A Hopf $R$-algebra, in this paper, will be a finitely generated projective $R$-module together with maps

$\mu: H \otimes H \rightarrow H$ (multiplication),

$i: R \rightarrow H$ (unit),

$\varepsilon: H \rightarrow R$ (counit),

$\Delta: H \rightarrow H \otimes H$ (comultiplication), and

$s: H \rightarrow H$ (antipode)

making $H$ into a Hopf $R$-algebra. As is customary, we write

$$
\mu\left(h_{1} \otimes h_{2}\right)=h_{1} h_{2}
$$


and we adopt the Sweedler notation

$$
\Delta(h)=\sum_{(h)} h_{(1)} \otimes h_{(2)}
$$

for comultiplication.

(1.2) The linear dual $H^{*}=\operatorname{Hom}_{R}(H, R)$ is a Hopf $R$-algebra with operations induced by duality; for example, for $f, g$ in $H^{*}$,

$$
\Delta^{*}(f)=f \cdot \mu, \quad \mu^{*}(f \otimes g)=(f \otimes g) \circ \Delta .
$$

The ${ }^{*}$ in $\Delta^{*}$ will often be suppressed when there is no danger of confusion.

(1.3) The augmentation ideal of $H$ is $\operatorname{ker}\{\varepsilon: H \rightarrow R\}$.

(1.4) The space of integrals $I$ of $H$ is defined by

$$
I=\left\{f \in H^{*} \mid g f=\varepsilon(g) f \text { for all } g \text { in } H^{*}\right\} .
$$

Since $H$ is finitely generated and projective as $R$-module, $I$ is nonzero, and, in fact, as $R$-modules, $H^{*} \cong H \otimes I[\mathbf{2 1}$, p. 98], so that $I$ is a rank one projective $R$-module.

(1.5) EXAMPLES. (a) If $G$ is a finite group, the group ring $R G$ is a Hopf algebra with $\varepsilon, \Delta, s$ defined by $\varepsilon(\sigma)=1, \Delta(\sigma)=\sigma \otimes \sigma, s(\sigma)=\sigma^{-1}$, and $I=R\left(\sum_{\sigma \in G} \sigma\right)$.

(b) $(R G)^{*}$ is a Hopf algebra. If $\left\{v_{\sigma}\right\} \subseteq(R G)^{*}$ is the dual basis to $\{\sigma \mid \sigma \in G\}$, then the $v_{\sigma}$ are pairwise orthogonal idempotents summing to 1 , and

$$
\begin{aligned}
& \Delta\left(v_{\sigma}\right)=\sum_{\tau} v_{\tau} \otimes v_{\tau^{-1} \sigma} \\
& \varepsilon\left(v_{\sigma}\right)=\delta_{1, \sigma} \quad(\text { Kronecker's delta) } \\
& s\left(v_{\sigma}\right)=v_{\sigma^{-1}}
\end{aligned}
$$

Here $I=R v_{1}$.

(c) Over rings of integers of algebraic number fields, group schemes of order $p$ have been classified by J. Tate and F. Oort [22]; locally, and in some cases globally these group schemes are represented by Hopf algebras which are free of rank $p$. When $p=2$ see also [17].

(1.6) Let $R$ be a perfect field of characteristic $p$, and $H$ be a Hopf $R$-algebra which is commutative and cocommutative. Then

$$
H^{*} \cong H_{1}^{*} \otimes_{R} H_{2}^{*},
$$

where $H_{1}^{*}$ is a separable $R$-algebra (cf. $\left[23\right.$, p. 47]) and $H_{2}^{*}$ is connected, that is, as algebra has the form

$$
H_{2}^{*}=R\left[h_{1}, \ldots, h_{n}\right] /\left\langle h_{1}^{p^{e_{1}}}, \ldots, h_{n}^{p^{e_{n}}}\right\rangle
$$

(where if $p=0, H^{*}=H_{1}^{*}$ ). For a proof, see Waterhouse [23, pp. 52, 112].

(1.7) Definition. A commutative $R$-algebra $S$ is an $H$-object if $S$ is a finitely generated projective $R$-module and a right $H$-comodule via a map $\alpha: S \rightarrow S \otimes H$ which is an $R$-algebra homomorphism. (Notation: $\alpha(s)=\sum_{(s)} s_{(0)} \otimes s_{(1)}$.)

(1.8) $S$ is an $H$-object if and only if $S$ is a left $H^{*}$-module via a module structure map $: H^{*} \otimes S \rightarrow S$ which is a measuring, in the sense that

$$
f \cdot(s t)=\sum_{(f)}\left(f_{(1)} \cdot s\right)\left(f_{(2)} \cdot t\right), \quad f \text { in } H ; s, t \text { in } S .
$$


The correspondence between $\alpha$ and · is as follows:

$$
f \cdot s=\sum_{(s)}\left\langle f, s_{(1)}\right\rangle s_{(0)},
$$

where $\langle$,$\rangle is the duality pairing H^{*} \otimes H \rightarrow R$; if $\left\{h_{i}, f_{i}\right\}_{i \in I}$ is a projective coordinate system for $H$ and $H^{*}$ so that

$$
h=\sum_{i \in I} f_{i}(h) h_{i}
$$

for all $h$ in $H$, then

$$
\alpha(s)=\sum_{i} f_{i} s \otimes h_{i} \quad(\text { cf. }[\mathbf{2 1}, \text { p. 36] }) .
$$

(1.9) If $S$ is an $H$-object, the fixed ring under the action of $H^{*}$ is

$$
S^{H^{*}}=\left\{s \in s \mid f \cdot s=\varepsilon(f) s \text { for all } f \text { in } H^{*}\right\} .
$$

When $H^{*}=R G$, an $H$-object $S$ is an $R$-algebra on which $G$ acts as a group of $R$-algebra automorphisms and $S^{H^{*}}=S^{G}$, the fixed ring under the action of $G$.

(1.10) The trivial $H$-object is $H$ itself, with $\alpha=\Delta$. Then $H^{*}$ acts on $H$ by

$$
f \cdot h=\sum_{(h)}\left\langle f, h_{(1)}\right\rangle h_{(0)} .
$$

(1.11) Suppose $S$ is an $H$-object via $\alpha$. Then $S$ is a Galois $H$-object if the map $\gamma: S \otimes S \rightarrow S \otimes H$ defined by $\gamma(s \otimes t)=\sum_{(t)} s t_{(0)} \otimes t_{(1)}$ is surjective. (By [16, 1.7] this is equivalent to $\gamma$ being an isomorphism.)

(1.12) ExAmples. (a) $S$ is a Galois $(R G)^{*}$-object if and only if $S$ is a Galois extension with group $G$ in the sense of $[\mathbf{6}]$.

(b) $S$ is a Galois $R G$-object if and only if $S=\sum_{\sigma \in G} S_{\sigma}$, where $S_{\sigma}$ are rank one projective $R$-modules, with

$$
S_{\sigma} S_{\tau}=S_{\sigma \tau} \quad \text { for all } \sigma, \tau \text { in } G .
$$

In particular, $S$ is fully graded in the sense of Dade [10].

(c) $H$ is a Galois $H$-object: the map $\gamma: H \otimes H \rightarrow H \otimes H$ defined by $\gamma(g \otimes h)=$ $(g \otimes 1) \Delta(h)=\sum g h_{(1)} \otimes h_{(2)}$ has an inverse $\theta([\mathbf{7}$, Proposition 9.1]),

$$
\theta(g \otimes h)=\sum g s\left(h_{(1)}\right) \otimes h_{(2)} \quad(s=\text { antipode }) .
$$

(d) Sources of examples of Galois $H$-objects, $H \neq R G,(R G)^{*}$, include Chase and Sweedler [7, p. 35, Example 4.11], Chase [5, Proposition 5.2], Kreimer [17], Hurley $[\mathbf{1 3}]$, and Childs $[\mathbf{8}, \mathbf{9}]$.

(1.13) Suppose $S$ is a Galois $H$-object. The smash product of $S$ and $H^{*}, D=$ $S \# H^{*}$, is defined to be $S \otimes H^{*}$ as $R$-module, with multiplication defined by

$$
(s \# h)(\bar{s} \# \bar{h})=\sum_{(h)} s\left(h_{(1)} \cdot \bar{s}\right) \# h_{(2)} \bar{h} .
$$

Then $D \cong \operatorname{End}_{R}(S)$. If $M$ is any left $D$ module, then $M$ is also an $H^{*}$-module and $M \cong S \otimes(I M)$ as $D$-module where $I$ is the space of integrals of $H^{*}$, via the map $s \otimes f \cdot m=s(f \cdot m)[\mathbf{7}$, p. 69].

From (1.13) we have immediately

(1.14) $H$ is a faithful $H^{*}$-module, 
and the useful

(1.15) Proposition. Suppose $S, T$ are $H$-objects and $\phi: S \rightarrow T$ is an $R$ algebra, $H^{*}$-module homomorphism. If $S$ is a Galois $H$-object and $I T=R$ where $I$ is the space of integrals of $H^{*}$, then $\phi$ is an isomorphism.

Proof of (1.15). View $T$ as a $\left(D=S \# H^{*}\right)$-module via $(s \# f) t=\phi(s) f t$ : that is, $S$ acts on $T$ via $\phi$. Then $T \cong S \otimes I T$ via $s \otimes f t \rightarrow \phi(s) f t$. If $I T=R$, then $S \cong S \otimes I T$ by $s \rightarrow s \otimes 1$, and the composite isomorphism $S \rightarrow S \otimes I T \rightarrow T$ is $\phi$.

2. Tame objects. Let $G$ be a finite group, $R$ the ring of integers of an algebraic number field $K, L$ a Galois extension field of $K$ with group $G$, and $S$ the ring of integers of $L$. Then $S \supset R$ is tame if for each prime ideal $p$ of $R$, the ramification index of $p$ in $S$ is relatively prime to the characteristic of $R / p$. This condition is equivalent to the condition that the trace map $\operatorname{tr}: S \rightarrow R, \operatorname{tr}(s)=\sum \sigma(s)$, is onto $[4$, p. 33].

When $R$ and $S$ are not necessarily rings of integers, and $G$ is a group of $R$-algebra automorphisms of $S$ with $S^{G}=R$, notions of tameness have been developed by Auslander and Rim [1] and A. D. Barnard [2]. Auslander and Rim assume $R$ is an integrally closed, local, Noetherian domain, and develop (without assuming a group $G$ of automorphisms) a generalization of the ramification index using multiplicity theory. Tameness is defined as in the classical case to be the condition that for all primes of $S$, the ramification index is prime to the residue field characteristic. Barnard works with general commutative rings and develops a theory of decomposition group, inertia group, and ramification group analogous to the classical theory for number fields [4, p. 33]. Tameness is defined as triviality of the ramification group at every prime, which again conforms to the classical definition.

Both Auslander/Rim and Barnard show that in their respective situations, $S$ $R$ is tame if and only if the trace map $\operatorname{tr}: S \rightarrow R$ is surjective.

If we translate these situations into Hopf algebra terms, we are considering $S$ to be an $H$-object for $H=(R G)^{*}$. Then $S^{G}=S^{R G}=S^{H^{*}}$.

It is easy to verify that the image of the trace map is $\left(\sum \sigma\right) S$ and $\sum \sigma$ generates the space of integrals for $H=R G$. Thus the statement that $\operatorname{tr}: S \rightarrow S^{R G}=R$ is surjective translates to $I S=S^{R G}=R$.

Note that for any $s, \operatorname{tr}(s)$ is in $R$, so $I S \subseteq S^{R G}$. More generally, the reader may easily verify (or see $[\mathbf{2 1}$, p. 203]):

(2.1) If $H$ is a Hopf algebra and $S$ an $H$-object, then $I S \subseteq S^{H^{*}}$.

The above observations motivate (c) of the next definition. Recall that in this paper both Hopf algebras and their objects are assumed to be finitely generated projective $R$-modules.

(2.2) Definition. Suppose $H$ is a Hopf $R$-algebra and $S$ is an $H$-object. Then $S$ is a tame $H$-object if

(a) $\operatorname{rank}_{R}(S)=\operatorname{rank}_{R}(H)$ as projective $R$-modules (this means locally at each prime ideal of $R$ if $S$ and $H$ do not have constant rank);

(b) $S$ is a faithful $H^{*}$-module; and

(c) $I S=S^{H^{*}}=R$.

In view of the preceding discussion it is clear that if $O_{L} \supset O_{K}$ is a tame extension of number rings, $O_{L}$ is a tame $O_{K} G^{*}$-object in our sense, and vice versa, conditions (a) and (b) being known to hold for number ring extensions. 
When $H^{*}=R G$ and $R \subset S$ are rings of integers, $S$ is a Galois extension of $R$ if no prime ideal of $R$ ramifies in $S$, and so Galois implies tame. That implication holds generally:

(2.3) Proposition. Let $H$ be a Hopf algebra and $S$ a Galois H-object. Then $S$ is tame.

Proof. That $S^{H^{*}}=R$ is [7, Theorem 7.6]. If $S$ is a Galois $H$-object, then for all primes $p$ of $R, S \otimes S \otimes R_{p} \cong S \otimes H \otimes R_{p}$ as $R_{p}$-modules; it is then clear that the local ranks agree. The faithfulness of $S$ follows from the isomorphism $S \# H^{*} \cong \operatorname{End}_{R}(S)$.

For condition (c), we view $S$ as a module over the smash product $D=S \# H^{*}$, $(s \# u) \cdot x=s(u \cdot x)$. Then by $(1.13), S=(I S) S$. But $S$ is finitely generated and projective so a routine Cramer's rule argument yields that $I S=R$ (or see the proof of (1.9) and (1.10) of [16]). It follows that $S$ is a tame $H$-object.

(2.4) COROllary. $H^{H^{*}}=R=I H$.

For $H$ is a Galois $H$-object $(1.12)(\mathrm{c})$.

We wish to show that $H$-objects with local normal bases are tame. Using Kreimer and Cook's result $[\mathbf{1 5}]$ that Galois $H$-objects have local normal bases, this will give a second proof of (2.3). We first need a "base change" lemma.

(2.5) LEMMA. (i) If $\tilde{R}$ is a flat extension of $R$ and $S$ is a tame $H$-object, then $S \otimes_{R} \tilde{R}$ is a tame $\left(H \otimes_{R} \tilde{R}\right)$-object.

(ii) If $\tilde{R}$ is faithfully flat and $S$ is an $H$-object such that $S \otimes \tilde{R}$ is a tame $(H \otimes \tilde{R})$ object, then $S$ is a tame $H$-object.

Proof. Set $\tilde{S}=S \otimes_{R} \tilde{R}, \tilde{H}=H \otimes_{R} R$, and $\tilde{H}^{*}=H^{*} \otimes_{R} \tilde{R}$ (unambiguous since $H$ is finitely generated projective over $R$ ).

(a) If $S, H$ are finitely generated and projective, then so are $\tilde{S}$ and $\tilde{H}$, and conversely if $\tilde{R}$ is faithfully flat $[3, \mathrm{I}, \S 3$, no. 6, Proposition 12].

If $\operatorname{rank}_{R} S=\operatorname{rank}_{R} H$ locally, then $\operatorname{rank}_{\tilde{R}} \tilde{S}=\operatorname{rank}_{\tilde{R}} \tilde{H}$ locally by the proof of $[\mathbf{3}$, II, $\S 5$, no. 4, Proposition 4]; for $\tilde{R}$ faithfully flat, the converse follows from the fact that every prime ideal of $R$ is the contraction of a prime ideal of $\tilde{R}[\mathbf{3}$, II, $\S 2$, no. 5 , Corollary 4 to Proposition 11].

(b) and (c) $S$ is a faithful $H^{*}$-module if and only if $\operatorname{ker}\left\{\beta^{*}: H^{*} \rightarrow \operatorname{End}_{R}(S)\right\}$ is zero. Also, $S^{H^{*}}=\operatorname{ker}\{\beta: S \rightarrow S \otimes H\}$ where $\beta=\alpha-\varepsilon_{1}, \varepsilon_{1}(s)=s \otimes 1$ [7, Proposition 10.1]. Thus the faithfulness property and the property $S^{H^{*}}=R$ are preserved under flat extension and reflected under faithfully flat extension. Finally $I_{H^{*} \otimes \tilde{R}}=I_{H^{*}} \otimes \tilde{R}$ if $\tilde{R}$ is $R$-flat since $I_{H^{*}}=\left(H^{*}\right)^{H^{*}}$; it follows that $I_{\tilde{H}^{*}} \tilde{S}^{*}=I_{H^{*}} S \otimes \tilde{R}$, and the property $I_{H^{*}} S=R$ is also preserved under flat extension and reflected under faithfully flat extension.

(2.6) COROLlary. The $H$-object $S$ is tame if and only if $S \otimes R_{p}$ is a tame $\left(H \otimes R_{p}\right)$-object for all primes $p$ of $R$.

PROOF. If $S$ is a tame $H$-object, then, since $R_{p}$ is $R$-flat, $S \otimes R_{p}$ is a tame $\left(H \otimes R_{p}\right.$ )-object for any prime $p$. The converse follows from the easily seen fact that if $S \otimes R_{p}$ is a tame $\left(H \otimes R_{p}\right)$-object for all $p$, then $S \otimes \prod_{p} R_{p}$ is a tame $\left(H \otimes \prod_{p} R_{p}\right.$ )-object, and $\prod_{p} R_{p}$ is $R$-faithfully flat. 
(2.7) THEOREM. Suppose $S$ is an $H$-object such that $S^{H^{*}}=R$ and $S \otimes R_{p} \simeq$ $H \otimes R_{p}$ as $\left(H^{*} \otimes R_{p}\right)$-module for all primes $p$ of $R$. Then $S$ is tame.

PROOF. In view of Corollary (2.6) we may without loss of generality assume $R$ is local and $S \simeq H$ as $H^{*}$-module.

Suppose $\phi: S \rightarrow H$ is the $H^{*}$-isomorphism. Then since $H$ satisfies conditions (a) and (b) and $\phi^{-1}$ must preserve these properties, $S$ satisfies (a) and (b). Since $\phi$ is an $H^{*}$-module homomorphism, $\phi(I S) \subseteq I H$; also, $\phi\left(S^{H^{*}}\right) \subseteq H^{H^{*}}$ since

$$
S^{H^{*}}=\left\{s \text { in } S \mid(f-\varepsilon(f)) s=0 \text { for all } f \text { in } H^{*}\right\} .
$$

Since $\phi$ is an isomorphism, the inclusions are equalities.

(2.8) Note. If $R$ is integrally closed, then the assumption in (2.7) that $S^{H^{*}}=$ $R$ may be dropped. Since $H$ is the trivial $H$-Galois object, $H^{H^{*}}=R$; by the measuring property, $S^{H^{*}} \supseteq R$. If $\phi: H \rightarrow S$ is an $H^{*}$-module isomorphism, then $\phi\left(S^{H^{*}}\right)=H^{H^{*}}=R$ so $\phi\left(1_{S}\right)=t$ for some $t$ in $R$. But then, since $\phi^{-1}$ is $R$-linear,

$$
1_{S}=\phi^{-1}(t)=t \phi^{-1}(1)
$$

in $S^{H^{*}}$. So $t$ is invertible in $S$. If $R$ is integrally closed, since $S$ is integral over $R$, then $t^{-1}=\phi^{-1}(1)$ is in $R$. But as $R$-module, $S^{H^{*}}=R \phi^{-1}(1)$, and so $S^{H^{*}} \subseteq R$.

(2.9) REMARK. Theorem 2.7 shows that the following three conditions making up the definition of tame $H$-object are necessary for the existence of local normal basis:

(a) equal ranks of $H$ and $S$,

(b) faithful $H$-action on $S$, and

(c) the space of integrals maps $S$ onto $R$, the fixed ring of $S$ under $H^{*}$.

The following two examples show that conditions (a) and (b) are independent of condition (c).

(2.10) The first example is due to A. D. Barnard [2, p. 283] and shows that it is possible to construct an $H$-object with $S^{H^{*}}=I S=R$ but which is not finitely generated as an $R$-module.

Let $R$ be a field $(\operatorname{char} \neq 2)$. As a set let

$$
S=\{a+p(x) \mid a \in R, p(x) \in R[x] \text { with only odd powers of } x\} .
$$

$S$ is a ring with the usual addition and multiplication given by $x^{\text {odd }} \cdot x^{\text {odd }}=0$, that is

$$
(a+p(x))(b+q(x))=a b+a q(x)+b p(x) .
$$

$S$ is an $R$-algebra via $a \mapsto a+0$. The group ring $R G, G$ of order 2 , acts on $S$ : if $\sigma$ generates $G$, then

$$
\sigma(a+p(x))=a-p(x) .
$$

It is easy to check that

$$
\sigma((a+p(x))(b+q(x)))=\sigma(a+p(x)) \sigma(b+q(x))
$$

so that the $R G$-action measures and $S$ is an $(R G)^{*}$-object. It is clear that $S^{G}=R$ and trace $\left(\frac{1}{2}\right)=1$ so $I S=S^{G}=R$. Hence condition (c) holds. It is also clear that $S$ is not finitely generated over $R$. So (a) fails, and we cannot have $S \cong H$ as $H^{*}$-module.

(2.11) For the second example, let $G$ be a finite group and $H$ the group ring $R G$. Then $H^{*}=R G^{*}=\bigoplus_{\sigma \in G} R v_{\sigma}$ as $R$-algebra with $\left\{v_{\sigma}\right\}_{\sigma \in G}$ mutually orthogonal 
idempotents. Let $S$ be an $H$-object such that $S^{H^{*}}=R$. We shall show condition (c) always holds but not condition (b).

Since $H^{*}=\bigoplus_{\sigma \in G} R v_{\sigma}$ as $R$-algebra, the action of $H^{*}$ on $S$ yields $S=\bigoplus_{\sigma \in G} v_{\sigma}$. $S$ as $H^{*}$-module, where $v_{\sigma} \cdot s=s$ for $s \in v_{\sigma} \cdot S$, and $v_{\tau} \cdot s=0$ for $\tau \neq \sigma$. Since $\varepsilon\left(v_{\sigma}\right)=\delta_{1, \sigma}$, it is straightforward to show that $I=R v_{1}$, and that $v_{1} \cdot S=S^{H^{*}}=I S$. In fact:

(2.12) Proposition. For $H=R G$, an $H$-object $S$ is tame if and only if $S^{H^{*}}=R$ and $S$ satisfies conditions (a) and (b) of (2.2).

To show that an $H$-object may not be a faithful $H^{*}$-module for $H=R G$, consider the following example.

Let $R$ be a field and $H=R(\mathbf{Z} / 3 \mathbf{Z})$. Let $S$ be the $R$-algebra

$$
S=\frac{R[x, y]}{\left\langle x^{2}, y^{2}, x y\right\rangle} \text {. }
$$

$S$ is an $H$-object via the map $\alpha: S \rightarrow S \otimes H$ given by

$$
\begin{aligned}
& \alpha(r)=r \otimes 1 \quad \text { for all } r \in R, \\
& \alpha(x)=x \otimes \sigma, \quad \alpha(y)=y \otimes \sigma,
\end{aligned}
$$

extended to $S$ by the requirement that $\alpha$ be an $R$-algebra map. This is well defined since

$$
\alpha\left(x^{2}\right)=x^{2} \otimes \sigma^{2}=0, \quad \alpha\left(y^{2}\right)=y^{2} \otimes \sigma^{2}=0, \quad \alpha(x y)=x y \otimes \sigma^{2}=0,
$$

so $S$ is an $H$-object. But, recalling that $f \cdot s=\sum_{(s)}\left\langle f, s_{(1)}\right\rangle s_{(0)}$, for $f \in H^{*}$ and $s \in S$, we see that $S$ is not a faithful $H^{*}$-module since

$$
v_{\sigma^{2}} \cdot r=\left\langle v_{\sigma^{2}}, 1\right\rangle r=0, \quad v_{\sigma^{2}} \cdot x=\left\langle v_{2}, \sigma\right\rangle x=0,
$$

and

$$
v_{\sigma^{2}} \cdot y=\left\langle v_{\sigma^{2}}, \sigma\right\rangle y=0
$$

so $v_{\sigma^{2}} \cdot S=0$.

3. Two special cases. In Theorem (2.7) we showed that $H$-objects with local normal basis are tame. This section establishes the converse for cocommutative Hopf algebras whose duals are separable or connected. We consider the separable case first.

(3.1) THEOREM. Let $H$ be a cocommutative Hopf $R$-algebra such that $H^{*}$ is separable. If $S$ is a tame $H$-object, then $S$ has local normal bases, i.e. $S \otimes R_{p} \cong R_{p}$ as $\left(H^{*} \otimes R_{p}\right)$-module for all primes $p$ of $R$.

This theorem applies, for example, if $\operatorname{dim} H$ is a unit of $R$, for then $H$ and $H^{*}$ are separable [22, Lemma 5]. Thus tame implies normal basis over fields of characteristic 0 .

The proof of this theorem makes use of the following lemma.

(3.2) LEMMA. Suppose $H$ is a cocommutative Hopf algebra over a local ring $R$ and $S$ is an $H$-object. Suppose $\tilde{R}$ is a faithfully flat extension of $R$. Then $S \simeq H$ as $H^{*}$-module if and only if $S \otimes \tilde{R} \cong H \otimes \tilde{R}$ as $\left(H^{*} \otimes \tilde{R}\right)$-module.

PROOF. If $S \cong H$ as $H^{*}$-modules, then $S \otimes \tilde{R} \cong H \otimes \tilde{R}$ as $(H \otimes \tilde{R})$-modules. Conversely, suppose $S \otimes \tilde{R} \cong H \otimes \tilde{R}$ as $\left(H^{*} \otimes \tilde{R}\right)$-modules. Now $H \otimes \tilde{R}$ is a Galois 
$(H \otimes \tilde{R})$-object, hence is a rank one projective $\left(H^{*} \otimes \tilde{R}\right)$-module by [15], as is, therefore, $S \otimes \tilde{R}$. If $\tilde{R}$ is faithfully flat over $R$, then $H^{*} \otimes \tilde{R}$ is faithfully flat over $H^{*}$. Thus $S$ and $H$ are rank one projective $H^{*}$-modules. Since $H^{*}$ is semilocal, the lemma follows.

Proof OF ThEOREM (3.1). We can assume $R$ is local by (2.6). Then both tameness and $S \simeq H$ are preserved and reflected by faithfully flat extensions (Lemmas (2.5) and (3.2)). Using [11, Lemma 2.8, p. 97], we may therefore assume in the proof of the theorem that since $H^{*}$ is separable, $H^{*}=R e_{1} \oplus R e_{2} \oplus \cdots \oplus R e_{n}$ as $R$-algebra with the $\left\{e_{i}\right\}$ mutually orthogonal idempotents.

Since $S$ is an $H$-object, the action of $H^{*}$ on $S$ yields $S \simeq \bigoplus_{i=1}^{n} S_{i}$ as $H^{*}$ module, where $S_{i}=e_{i} \cdot S$. Now $S$ is free so each $S_{i}$ is a projective $R$-module which is free since $R$ is local. Since $S$ is tame, $\operatorname{dim}_{R} S=n$ and each $S_{i}$ is nonempty by faithfulness. Hence for each $i, S_{i} \simeq R$ as $R$-module. Let $s_{i}$ generate $S_{i}$ over $R$. Then $S \simeq \bigoplus_{i=1}^{n} R s_{i}$ as $H^{*}$-module with $e_{i} \cdot s_{j}=\delta_{i, j} s_{j}$. In particular, since $H$ is a tame $H$-object $H \simeq \bigoplus_{i=1}^{n} R x_{i}$ as $H^{*}$-module with $e_{i} x_{j}=\delta_{i, j} x_{j}$.

It is then apparent that the map $\phi: S \rightarrow H$ given by $\phi\left(s_{i}\right)=x_{i}$ is an $H^{*}$-module isomorphism. This establishes Theorem (3.1).

Now we consider $H$-objects when $H^{*}$ is connected (cf. (1.6)).

(3.3) THEOREM. Suppose $R$ is a commutative ring and $H$ is a cocommutative Hopf algebra such that, as R-algebra,

$$
H^{*}=\frac{R\left[f_{1}, f_{2}, \ldots, f_{n}\right]}{\left\langle f_{1}^{p^{1}}, f_{2}^{p^{e_{2}}}, \ldots, f_{n}^{p^{e_{n}}}\right\rangle}
$$

for some prime $p$, where $\varepsilon\left(f_{i}\right)=0$ for all $i=1, \ldots, n$. Suppose $S$ is an $H$-object, not necessarily finitely generated and projective as $R$-module, such that $I S=S^{H^{*}}=R$. Then $S$ is a Galois $H$-object.

The hypothesis on $S$ is (c) of the definition of tameness. Thus this theorem implies that when $H^{*}$ is connected, an $H$-object $S$ is tame if and only if $S$ is Galois if and only if $I S=S^{H^{*}}=R$.

PROOF. Kreimer and Takeuchi [16] proved that an $H$-object $S$, not a priori finitely generated and projective as $R$-module, is a Galois $H$-object (and hence is finitely generated and projective as $R$-module) if the left $S$-module map $\gamma: S \otimes S \rightarrow$ $S \otimes H$ given by

$$
\gamma(s \otimes t)=\sum_{(t)} s t_{(0)} \otimes t_{(1)}
$$

is surjective.

If $\left\{x_{i}, f_{i}\right\}_{i \in H}$ is a dual basis sytem for $H, H^{*}$, then $\alpha$ can be recovered from the $H^{*}$-module action on $S$ by

$$
\alpha(s)=\sum_{i} f_{i} \cdot s \otimes x_{i} \quad(\text { see }(1.8))
$$

so

$$
\gamma(s \otimes t)=\sum_{i} s f_{i} \cdot t \otimes x_{i}
$$


Thus the set $\left\{1 \otimes x_{i}\right\}$ is an $S$-basis for $S \otimes H$, and to show $S$ is Galois we find, for each $i$, a set $\left\{\left(a_{K}, b_{K}\right)\right\} \subseteq S \times S$ such that

$$
\gamma\left(\sum_{K} a_{K} \otimes b_{K}\right)=1 \otimes x_{i}
$$

Now $H^{*}$ has $R$-basis $\left\{f_{1}^{j_{1}} f_{2}^{j_{2}} \cdots f_{n}^{j_{n}} \mid 0 \leq j_{i}<p^{e_{i}}\right.$ for $\left.i=1, \ldots, n\right\}$, and we have

(3.4) The space of integrals $I$ of $H^{*}$ is generated by the element

$$
\phi=f_{1}^{p^{e_{1}}-1} f_{2}^{p^{e_{2}}-1} \cdots f_{n}^{p^{e_{n}}-1} .
$$

To see (3.4), note that for any integral $\psi$ of $I$ and any $i, f_{i} \psi=\varepsilon\left(f_{i}\right) \psi=0$. Thus $\psi$ must be a multiple of $\phi$.

Since $I S=R$, there exists some $z \in S$ such that $\phi z=1$.

(3.5) We adopt the following multinomial notation.

$$
\text { If }
$$

$$
F=\left(f_{1}, f_{2}, \ldots, f_{n}\right), \quad J=\left(j_{1}, j_{2}, \ldots, j_{n}\right),
$$

then

$$
F^{J}=f_{1}^{j_{1}} f_{2}^{j_{2}} \cdots f_{n}^{j_{n}}, \quad|J|=\sum_{i=1}^{n} j_{i}
$$

and

$$
F^{\bar{J}}=f_{1}^{p^{e_{1}}-j_{1}-1} f_{2}^{p^{e_{2}}-j_{2}-1} \cdots f_{n}^{p^{e_{n}}-j_{n}-1} .
$$

In this notation, our basis for $H^{*}$ becomes

$$
\left\{F^{J} \mid 0 \leq j_{i}<p^{e_{i}}, i=1, \ldots, n\right\} .
$$

Let $\left\{X^{J} \mid 0 \leq j_{i}<p^{e_{i}}, i=1, \ldots, n\right\}$ denote the basis of $H$ dual to that for $H^{*}$.

To show that $S$ is a Galois $H$-object, we shall find for every $J$ a set $\left\{\left(a_{K}, b_{K}\right)\right\} \subseteq$ $S \times S$ such that

$$
\gamma\left(\sum_{K} a_{K} \otimes b_{K}\right)=\sum_{L}\left(\sum_{K} a_{K}\left(F^{L} \cdot b_{K}\right)\right) \otimes X^{L}=1 \otimes X^{J},
$$

where the sum over $L$ is over all elements of the basis of $H^{*}$; that is:

(3.6) Given any $J$ such that $0 \leq j_{i}<p^{e_{i}}$ for all $i$, there exists $\left\{\left(a_{K}, b_{K}\right)\right\} \subseteq S \times S$ such that

$$
\sum_{K} a_{K}\left(F^{J} \cdot b_{K}\right)=1, \quad \sum_{K} a_{K}\left(F^{H} \cdot b_{K}\right)=0 \text { for all } H \neq J .
$$

We define $a_{K}, b_{K}$ as follows: for all $K=\left(k_{1}, \ldots, k_{n}\right), 0<k_{i} \leq p^{e_{i}}$, let $b_{K}=$ $F^{\bar{K}} \cdot z\left(F^{\bar{K}}\right.$ as defined above $)$. We define $a_{K}$ as follows:

$$
\begin{array}{ll}
a_{K}=0 & \text { if }|K| \geq|J|, K \neq J \\
a_{J}=1, & \\
a_{K}=-\sum_{|L|>|K|} a_{L}\left(F^{K} b\right) & \text { if }|K|<|J| .
\end{array}
$$

Observe that:

(3.7) For all $K$ such that $|K| \geq|J|, K \neq J$, and all $H$,

$$
a_{K}\left(F^{H} \cdot b_{K}\right)=0 \quad \text { since } a_{K}=0 \text {. }
$$


(3.8) For all $H, K$ such that $|H| \geq|K|, H \neq K$,

$$
F^{H} \cdot b_{K}=\left(F^{H} F^{\bar{K}}\right) \cdot z=0
$$

since for some $i, f^{p^{e_{i}}}=0$ is a factor of $F^{H} F^{\bar{K}}$.

(3.9) $F^{K} \cdot b_{K}=\left(f_{1}^{p^{e_{1}}-1} \cdots f_{n}^{p^{e_{n}}-1}\right) \cdot z=1$ for all $K$.

To show that

$$
\sum_{K} a_{K}\left(F^{H} \cdot b_{K}\right)= \begin{cases}1 & \text { if } H=J \\ 0 & \text { otherwise }\end{cases}
$$

we have from (3.7) that

$$
\sum_{K} a_{K}\left(F^{H} \cdot b_{K}\right)=a_{J}\left(F^{H} \cdot b_{J}\right)+\sum_{|K|<|J|} a_{K}\left(F^{H} \cdot b_{K}\right) .
$$

Case 1. When $|H| \geq|J|$ but $H \neq J,(3.8)$ and (3.10) imply that

$$
\sum_{K} a_{K}\left(F^{H} \cdot b_{K}\right)=0
$$

Case 2. When $H=J,(3.8)$ and (3.10) imply that

$$
\sum_{|K|<|J|} a_{K}\left(F^{J} \cdot b_{K}\right)=0
$$

so $\sum_{K} a_{K}\left(F^{J} \cdot b_{K}\right)=a_{J}\left(F^{J} \cdot b_{J}\right)=1$ by (3.9) and the definition of $a_{J}$.

Case 3. When $|H|<|J|$,

$$
\sum_{K} a_{K}\left(F^{H} \cdot b_{K}\right)=\sum_{\substack{|H| \geq|K| \\ H \neq K}} a_{K}\left(F^{H} \cdot b_{K}\right)+a_{H}\left(F^{H} \cdot b_{H}\right)+\sum_{|H|<|K|} a_{K}\left(F^{H} \cdot b_{K}\right) .
$$

The first summand $=0$ by (3.8). The second summand $=a_{H}$ by (3.9) but

$$
a_{H}=-\sum_{|K|>|H|} a_{K}\left(F^{H} \cdot b_{K}\right)
$$

so $\sum_{K} a_{K}\left(F^{H} \cdot b_{K}\right)=0$.

This establishes (3.6). It follows that $\gamma$ is surjective and $S$ is a Galois $H$-object, completing the proof of Theorem (3.3).

It follows that $S$ is finitely generated and projective over $R$ by $[16,(1.7)]$. In fact, the reader may verify that if $z \in S$ satisfies $\phi z=1$, then $\left\{F^{J} \cdot z\right\}$ is an $R$-basis of $S$. Thus the map $1 \mapsto z$ yields an $H^{*}$-isomorphism $H^{*} \rightarrow S$, and $S$ has a normal basis (cf. also [16, Proposition 2.7]).

4. Normal bases over perfect fields. In this section we put together the results from $\S \S 2$ and 3 to characterize, over a perfect field, those $H$-objects with normal basis when $H$ is commutative and cocommutative.

(4.1) THEOREM. Suppose $H$ is a Hopf $R$-algebra, $R$ a commutative ring, such that $H^{*}=H_{1}^{*} \otimes H_{2}^{*}$, where $H_{1}^{*}$ is commutative and separable (as in (3.1)) and $H_{2}^{*}$ is connected (as in (3.3)). Then an $H$-object $S$ is tame if and only if $S$ has local normal bases.

ProOF. The "if" part is Theorem 2.7. 
For the converse, let $S$ be a tame $H$-object. Let $H_{1}^{*}$ act by restriction on $S^{H_{2}^{*}}$, and $H_{2}^{*}$ on $S^{H_{1}^{*}}$. Then the measuring property is inherited so that $S^{H_{2}^{*}}$ is an $H_{1}$-object, and $S^{H_{1}^{*}}$ an $H_{2}$-object (except that we must check finite generation and projectivity over $R$ ). We shall show that $S \cong S^{H_{2}^{*}} \otimes S^{H_{1}^{*}}, S^{H_{1}^{*}} \cong H_{2}$, and $S^{H_{2}^{*}} \cong H_{1}$ locally, as $H^{*}-, H_{2}^{*}$, and $H_{1}^{*}$-modules, respectively, from which it will follow that as $H^{*}$-modules, locally $S \cong H_{1} \otimes H_{2}=H$.

One verifies easily that $R=S^{H^{*}}=S^{H_{1}^{*} \otimes H_{2}^{*}}=\left(S^{H_{1}^{*}}\right)^{H_{2}^{*}}=\left(S^{H_{2}^{*}}\right)^{H_{1}^{*}}$, and that $I_{H^{*}}=I_{H_{1}^{*}} \otimes I_{H_{2}^{*}}$

We have

$$
R=I_{H^{*}} S=I_{H_{2}^{*}}\left(I_{H_{1}^{*}} S\right) \subseteq I_{H_{2}^{*}}\left(S^{H_{1}^{*}}\right) \subseteq\left(S^{H_{2}^{*}}\right)^{H_{1}^{*}}=R .
$$

Since $H_{2}^{*}$ is connected, by (3.3) $S^{H_{1}^{*}}$ is a Galois $H_{2}$-object and $S^{H_{1}^{*}} \cong H_{2}$ as $H_{2}^{*}$-objects. In particular, $S^{H_{1}^{*}}$ is finitely generated and projective over $R$.

Now $S$ is a left $D$-module, $D=S^{H_{1}^{*}} \# H_{2}^{*} \cong \operatorname{End}_{R}\left(S^{H_{1}^{*}}\right)$. By (1.15) $S \cong$

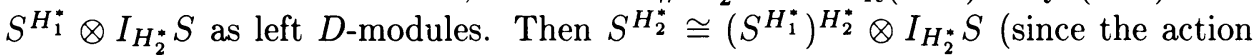
of $D$ on $S$ is via its action on $S^{H_{1}^{*}}$ ), so $S^{H_{2}^{*}}=I_{H_{2}^{*}} S$. Thus $S \cong S^{H_{1}^{*}} \otimes S^{H_{2}^{*}}$. The isomorphism is given by the multiplication in $S$, and this is easily seen to be an $H^{*}$-module map. We must show that $S^{H_{2}^{*}}$ is a tame $H_{1}^{*}$-object.

First, since $S^{H_{1}^{*}}$ is $R$-projective, $R$ is an $R$-module direct summand of $S^{H_{1}^{*}}$, so $S^{H_{2}^{*}} \cong R \otimes S^{H_{2}^{*}}$ is an $R$-direct summand of $S$. Since $S$ is $R$-projective, so is $S^{H_{2}^{*}}$.

Then $\operatorname{rank}_{R} S^{H_{2}^{*}}=\operatorname{rank}_{R} H_{1} \operatorname{since} \operatorname{rank} S=\operatorname{rank} H$ and $\operatorname{rank} S^{H_{1}^{*}}=\operatorname{rank} H_{2}$. Also $S^{H_{2}^{*}}$ is a faithful $H_{1}^{*}$-module since $S$ is a faithful $H$-module; and

$$
R=I_{H^{*}} S=I_{H_{1}^{*}}\left(I_{H_{2}^{*}} S\right) \subseteq I_{H_{1}^{*}}\left(S^{H_{2}^{*}}\right) \subseteq\left(S^{H_{2}^{*}}\right)^{H_{1}^{*}}=R .
$$

Thus $S^{H_{2}^{*}}$ is a tame $H_{1}$-object. By $(3.1) S^{H_{2}^{*}}$ has local normal basis. This completes the proof.

(4.2) COROLlaRY. Suppose $R$ is a perfect field and $H$ a commutative, cocommutative Hopf algebra over $R$. Then an $H$-object $S$ is tame if and only if $S \simeq H$ as $H^{*}$-module.

PROOF. This follows immediately from (4.1) and the decomposition of (1.6).

5. Local normal bases over integral domains. Corollary (4.2) established a criterion for the existence of a normal basis for an $H$-object $S, H$ commutative and cocommutative, over a perfect field. In case the field has characteristic 0 , Theorem (3.1) applies whenever $H$ is cocommutative.

In this section we consider the situation where $R$ is an integral domain with quotient field $K, H$ is a Hopf $R$-algebra, and $S$ is an $H$-object (both finitely generated and projective as $R$-modules). We suppose that $S \otimes K$ is an $(H \otimes K)$-object with normal basis, and seek criteria for $S$ to be an $H$-object with local normal bases.

Since $H$ is locally isomorphic to $H^{*}$ as $H^{*}$-module, an obvious necessary condition is that $S$ be $H^{*}$-projective. In the classical situation, $H^{*}=R G$, surjectivity of the trace map $S \rightarrow R$ implies projectivity (cf. [20, Lemma 20]). So also here.

(5.1) THEOREM. Let $R$ be a commutative ring, $H$ be an $R$-Hopf algebra which is a finitely generated projective $R$-module, and $S$ an $R$-algebra which is an $H$ object and a finitely generated projective $R$-module. Let $I$ be the space of integrals of $H^{*}$. If $I S=R$, then $S$ is $H^{*}$-projective. 
ProOF. Since $S$ is $H^{*}$-projective if and only if $S_{p}$ is $H_{p}^{*}$-projective for every prime ideal $p$ of $R$, we can assume $R$ is local. In that case, $I$ is free of rank one over $R, I=R \phi$ for some integral $\phi$, and the condition $I S=R$ becomes the condition that there exists $z$ in $S$ so that $\phi(z)=1$.

Since $S$ is $R$-projective, $H^{*} \otimes S$, with $H^{*}$-action on $H^{*}$, is $H^{*}$-projective.

Let $\mu: H^{*} \otimes S \rightarrow S$ be the $H^{*}$-action on $S$. With $H^{*}$-action on $H^{*} \otimes S$ via that on $H^{*}, \mu$ is an $H^{*}$-module map.

Let $\lambda: S \rightarrow H^{*} \otimes S$ be defined by

$$
\lambda(x)=\sum_{(\phi)} \phi_{(1)} \otimes z\left(\phi_{(2)}^{s} x\right) .
$$

Then

$$
\begin{aligned}
\mu \lambda(x) & =\sum_{(\phi)} \phi_{(1)}\left(z \cdot \phi_{(2)}^{s} x\right)=\sum_{(\phi)}\left(\phi_{(1)} \cdot z\right)\left(\phi_{(2)} \phi_{(3)}^{s} x\right) \\
& =\sum_{(\phi)}\left(\phi_{(1)} \cdot z\right) \varepsilon\left(\phi_{(2)}\right) x=\sum_{(\phi)}\left(\phi_{(1)} \varepsilon\left(\phi_{(2)}\right) z\right) x \\
& =\phi(z) x=x .
\end{aligned}
$$

So $\lambda$ is injective and splits $\mu$.

To show that $S$ is $H^{*}$-projective, it suffices to show that $\lambda$ is an $H^{*}$-module map, that is, $f \cdot \lambda(x)=\lambda(f \cdot x)$.

We thank the referee for pointing out the following direct argument of Sweedler [2, p. 104]:

For $f \in H^{*}$ we have $f=(1 \otimes \varepsilon) \Delta f=\sum_{(f)} \varepsilon\left(f_{(2)}\right) f_{(1)}$. Hence

$$
\begin{aligned}
\sum f \phi_{(1)} \otimes \phi_{(2)}^{s} & =\sum_{(\phi)(f)} \varepsilon\left(f_{(2)}\right) f_{(1)} \phi_{(1)} \otimes\left(\phi_{(2)}^{s}\right) \\
& =\sum_{(\phi)(f)} f_{(1)} \phi_{(1)} \otimes \varepsilon\left(f_{(2)}\right) \phi_{(2)}^{s} \\
& =\sum_{(1)} \phi_{(1)} \otimes \phi_{(2)}^{s} f_{(2)}^{s} f_{(3)} \\
& =\sum_{(1)} \phi_{(1)} \otimes\left(f_{(2)} \phi_{(2)}\right)^{s} f_{(3)} \\
& =\sum_{(f)}((1 \otimes s) \Delta)\left(f_{(1)} \phi\right)\left(1 \otimes f_{(2)}\right) \\
& =\sum_{(f)}((1 \otimes s) \Delta)\left(\varepsilon\left(f_{(1)}\right) \phi\right)\left(1 \otimes f_{(2)}\right) \\
& =(1 \otimes s) \Delta(\phi) \sum_{(f)}\left(1 \otimes \varepsilon\left(f_{(1)}\right) f_{(2)}\right) \\
& =(1 \otimes s) \Delta(\phi)(1 \otimes f)=\sum_{(\phi)} \phi_{(1)} \otimes \phi_{(2)}^{s} f .
\end{aligned}
$$


Hence

$$
\begin{aligned}
f(\lambda(x)) & =\sum_{(\phi)} f \phi_{(1)} \otimes z\left(\phi_{(2)}^{s} x\right) \\
& =(1 \otimes z)\left(\left(\sum f \phi_{(1)} \otimes \phi_{(2)}^{s}\right)(1 \otimes x)\right) \quad\left(1 \in H^{*}\right) \\
& =(1 \otimes z)\left(\left(\sum \phi_{(1)} \otimes \phi_{(2)}^{s} f\right)(1 \otimes x)\right) \\
& =\sum_{(\phi)} \phi_{(1)} \otimes z\left(\phi_{(2)}^{s}(f(x))=\lambda(f(x)) .\right.
\end{aligned}
$$

Thus $\lambda$ is an $H^{*}$-module map, as was to be shown. Hence $S$ is $H^{*}$-projective, since it is an $H^{*}$-direct summand of the $H^{*}$-projective module $H^{*} \otimes S$.

(5.2) TheOrem. Suppose $R$ is a domain with quotient field $K, H^{*}$ is a commutative Hopf $R$-algebra, and $S$ is an $H$-object. Let $L=S \otimes K$. If $S$ is a projective $H^{*}$-module and $L$ has a normal basis as an $H \otimes K$-object, then $S$ has local normal bases as an $H$-object.

PROOF. We may clearly assume that $R$ is a local ring. Write $H^{*}=\sum_{i} H^{*} e_{i}$, where $1=\sum_{i} e_{i}$, and the $e_{i}$ are indecomposable pairwise mutually orthogonal idempotents. Set $H^{*} e_{i}=I_{i}$, indecomposable $H^{*}$-modules. Let $S=J_{1} \oplus J_{2} \oplus \cdots \oplus J_{r}$ and $H=Y_{1} \oplus Y_{2} \oplus \cdots \oplus Y_{x}$ be direct sums of indecomposable $H^{*}$-modules.

We show $S \cong H$ as $H^{*}$-modules by showing, by induction on $r$ :

(5.3) If $J=J_{1} \oplus \cdots \oplus J_{r}$ and $Y=Y_{1} \oplus \cdots \oplus Y_{s}$ are two projective $H^{*}$-modules, direct sums of indecomposable $H^{*}$-modules, and $J \otimes K \cong Y \otimes K$ as $\left(H^{*} \otimes K\right)$ modules, then $J \cong Y$.

The proof is obvious for $r=1$.

For $r>1$, since $J$ is a projective $H^{*}$-module, $J_{r} \cong I_{i}=e_{i} H^{*}$ for some $i$. Then $e_{i} J \neq 0$, so $e_{i}(J \otimes K) \neq 0$. Since $J \otimes K \cong Y \otimes K, e_{i}(Y \otimes K) \neq 0$, so $e_{i}(Y) \neq 0$. But $e_{i}(Y)=0$ if $Y_{j} \not I_{i}$ for all $j$. So some $Y_{j} \cong I_{i} \cong J_{r}$.

Suppose $Y_{s} \cong J_{r}$. Write $J=J^{\prime} \oplus J_{r}, Y=Y^{\prime} \oplus Y_{s}$. Then

$$
J \otimes K \cong\left(J^{\prime} \otimes K\right) \oplus\left(J_{r} \otimes K\right)
$$

and

$$
Y \otimes K \cong\left(Y^{\prime} \otimes K\right) \oplus\left(Y_{s} \otimes K\right) .
$$

By the Krull-Schmidt Theorem $[\mathbf{1 9}$, p. 88]

$$
J^{\prime} \otimes K \cong Y^{\prime} \otimes K \text {. }
$$

By induction, $J^{\prime} \cong Y^{\prime}$. Since $J_{r} \cong Y_{s}$, we obtain $J \cong Y$, completing the proof of (5.3) and Theorem (5.2).

(5.4) THEOREM. Let $R$ be an integral domain with quotient field $K, H$ a cocommutative Hopf $R$-algebra which is a finitely generated projective $R$-module, and $S$ an $R$-algebra, finitely generated and projective as $R$-module, and an $H$-object such that $S^{H^{*}}=R$.

If $S$ has local normal bases, then $S$ is a tame $H$-object. Conversely, if $S$ is a tame $H$-object, then $S$ has local normal bases whenever

(a) $K$ is perfect and $H$ is commutative, or 
(b) $H \otimes K$ is separable.

Condition (b) holds if char $K=0$ or char $K$ is relatively prime to $\operatorname{dim}_{K}(H \otimes K)$.

PROOF. That local normal bases implies tame is Theorem (2.7).

For the converse, since $K$ is $R$-flat, if $S$ is a tame $H$-object, then $S \otimes K$ is a tame $(H \otimes K)$-object, and $S$ is a tame $H$-object for all prime ideals $p$ of $R$, both by Lemma (2.5)(i). So we may assume $R$ is a local domain. If $K$ is perfect, and $H$ is commutative, or if $H \otimes K$ is separable, we have $S \otimes K \cong H \otimes K$ as $\left(H^{*} \otimes K\right)$ modules, by Theorem (4.2), resp. (3.1). Since $S$ is tame, $S$ is $H^{*}$-projective by Theorem (5.1), and so by Theorem (5.2), $S$ has a normal basis. That completes the proof.

(5.5) REMARK. If $R$ is the ring of integers of a number field $K$, there are many examples of extensions $L \supset K$ whose ring of integers $S$ is a tame $H$-object for some Hopf algebra $H$. For example, if $K=Q, L=Q(\sqrt{m}), G=\operatorname{Gal}(L / K), R=\mathbf{Z}$, and $m \equiv 2$ or $3(\bmod 4)$, then $S$ is a tame $R G$-object, but is Galois only when $m=-1$. A characterization of those Kummer extensions $L \supset K$ of prime degree with Galois group $G$ such that the order $A$ over $R$ in $K G, A=\{\alpha \in K G \mid \alpha S \subseteq S\}$ (cf. [18 or 12, p. 251]) is a Hopf algebra (of the kind studied in [22]) and $S$ is a tame $A^{*}$-object, is given in $[\mathbf{9}]$. For those extensions, Theorem (5.4) guarantees a reasonable local description of $S$, with the aid of the characterizations of [14].

\section{REFERENCES}

1. M. Auslander and D. Rim, Ramification index and multiplicity, Illinois J. Math. 7 (1963), $566-581$.

2. A. D. Barnard, Commutative rings with operators (Galois theory and ramification), Proc. London Math. Soc. (3) 28 (1974), 274-290.

3. N. Bourbaki, Algèbre commutative, Chapitres I and II, Hermann, Paris, 1961.

4. J. Cassels and A. Frohlich (eds.), Algebraic number theory, Thompson, Washington, D. C., 1967.

5. S. Chase, Infinitesimal group scheme actions on finite field extensions, Amer. J. Math. 98 (1976), 441-480.

6. S. Chase, D. Harrison and A. Rosenberg, Galois theory and Galois cohomology over a commutative ring, Mem. Amer. Math. Soc. No. 52 (1965), 15-33.

7. S. Chase and M. Sweedler, Hopf algebra and Galois theory, Lecture Notes in Math., vol. 97, Springer-Verlag, Berlin and New York, 1969.

8. L. Childs, Azumaya algebras over number rings as smash products (preprint).

9. __ Taming wild extensions with Hopf algebras (preprint).

10. E. Dade, The equivalence of various generalizations of group rings and modules, Math. Z. 181 (1982), 335-344.

11. F. DeMeyer and E. Ingraham, Separable algebras over commutative rings, Lecture Notes in Math., vol. 181, Springer-Verlag, Berlin and New York, 1971.

12. A. Frohlich, Galois module structure of algebraic integers, Springer-Verlag, Berlin and New York, 1983.

13. S. Hurley, Tame and Galois Hopf objects with normal bases, Thesis, SUNY at Albany, 1984.

14. __ Galois objects with normal bases for free Hopf algebras or prime degree, J. Algebra (to appear).

15. H. F. Kreimer and P. Cook, Galois theories and normal bases, J. Algebra 43 (1976), 115-121.

16. H. Kreimer and M. Takeuchi, Hopf algebras and Galois extensions of an algebra, Indiana Univ. Math. J. 30 (1981), 675-692. 
17. H. F. Kreimer, Quadratic Hopf algebras and Galois extensions, Contemp. Math. 13 (1981), $353-361$.

18. H. W. Leopoldt, Uber die Hauptordnung der ganzen Elemente eines abelschen Zahlkorpers, J. Reine Angew. Math. 201 (1959), 119-149.

19. I. Reiner, Maximal orders, Academic Press, London, 1975.

20. J.-P. Serre, Representations linears des groupes fini, Hermann, Paris, 1971.

21. M. Sweedler, Hopf algebras, Benjamin, New York, 1969.

22. J. Tate and F. Oort, Group schemes of prime order, Ann. Sci. École Norm. Sup. (4) 3 (1970), 1-21.

23. W. Waterhouse, Introduction to affine group schemes, Springer-Verlag, Berlin and New York, 1979.

Department of Mathematics, State University of NeW York at Albany, ALBANY, NEW YORK 12222

Department of Mathematics, Siena College, Loudonville, New York 12211 